\title{
Tungsten Microstructural Results from the Gadolinium-Shielded 19J Irradiation Experiment
}

\author{
Chad M. Parish ${ }^{1, *}$, Lauren Garrison ${ }^{1}$, Eric Lang ${ }^{2}$, Jean-Paul Allain ${ }^{2}$, and Yutai Katoh ${ }^{1}$ \\ 1. Materials Science and Technology Division, Oak Ridge National Laboratory, Oak Ridge, TN, USA \\ 2. Nuclear, Plasma, and Radiological Engineering, University of Illinois, Urbana, IL, USA \\ * Corresponding author: parishcm@ornl.gov
}

Magnetic fusion energy (MFE) reactor concepts, such as the international ITER project, require plasmafacing materials (PFMs) that can withstand a harsh service environment that includes high-flux $14 \mathrm{MeV}$ neutrons, MW/m $/ \mathrm{m}^{2}$ thermal loads, and particles fluxes of $10^{22}$ ions $/ \mathrm{m}^{2} \mathrm{sec}$ or more of fuel $\left({ }^{2} \mathrm{H}+{ }^{3} \mathrm{H}\right)$ and ash $\left({ }^{4} \mathrm{He}\right)$, as well as thermal and mechanical shocks from plasma disruptions.

No known material can withstand the environment envisioned for eventual power-station-scale MFE reactors, but tungsten (W) is considered the leading candidate for future development at present. Unfortunately, no high-flux neutron sources with fusion-prototypic spectra are available at present, so fission neutrons must be used to perform irradiation experiments. Light-water-moderated spectra, such as at the ORNL High Flux Isotope Reactor (HFIR), have significant thermal-neutron contributions that drive excess transmutation of $\mathrm{W} \rightarrow \mathrm{Re} \rightarrow \mathrm{Os}$, compared to a fusion-like spectrum. This results in excessive intermetallic precipitation [1,2]. As such, the recent "19J" irradiation campaign was undertaken in the Fusion Materials program at ORNL, with collaboration from University of Illinois. In this experiment, irradiation capsules were constructed with gadolinium $(\mathrm{Gd})$ shields, which reduced the thermal neutron to fast neutron flux ratio by $\approx 10 \times$. We hypothesized, based on neutronics calculations, that this will result in a (very roughly) $\sim 5 \times$ reduction in transmutation per dpa, and therefore significantly less radiation-induced segregation (RIS) and radiation-induced precipitation (RIP) of intermetallic particles than in W samples irradiated unshielded. In this paper, we report preliminary experiments on these shielded-capsule specimens and compare their microstructures to the unshielded experiments.

EBSD (Tescan MIRA3 FEG-SEM+Oxford Symmetry CMOS EBSD) indicates little or no grain growth under irradiation at $500^{\circ} \mathrm{C}$ or $800^{\circ} \mathrm{C}$, Figure 1. Bright-field TEM (Talos F200X) indicates that void formation and intermetallic precipitation has occurred under irradiation, Figure 2. X-ray mapping in STEM, Figure 3, indicates grain boundary and possibly intragranular precipitation is underway. Experiments are ongoing to quantify the relative fractions of precipitates between unshielded and Gdshielded irradiated specimens, and to compare the mechanical properties and relate these changes to the microstructures. [3]

[1] X. Hu et al., J. Nuclear Mater. V480 (2016) p. 235.

[2] T. Koyanagi, J. Nucl. Mater. V490 (2017) p. 66.

[3] Research supported of US Department of Energy, Office of Science, Fusion Energy Sciences (including an Early Career Award), under contract number DE-AC05-00OR22725. A portion of this research used resources at the High Flux Isotope Reactor, a DOE Office of Science User Facility operated by the Oak Ridge National Laboratory. This work is partially supported by US DOE Contract No. DE-SC0014267. This research was supported in part by an appointment to the Oak Ridge National Laboratory NESLS Program, sponsored by the U.S. Department of Energy and administered by the Oak Ridge Institute for Science and Education. 

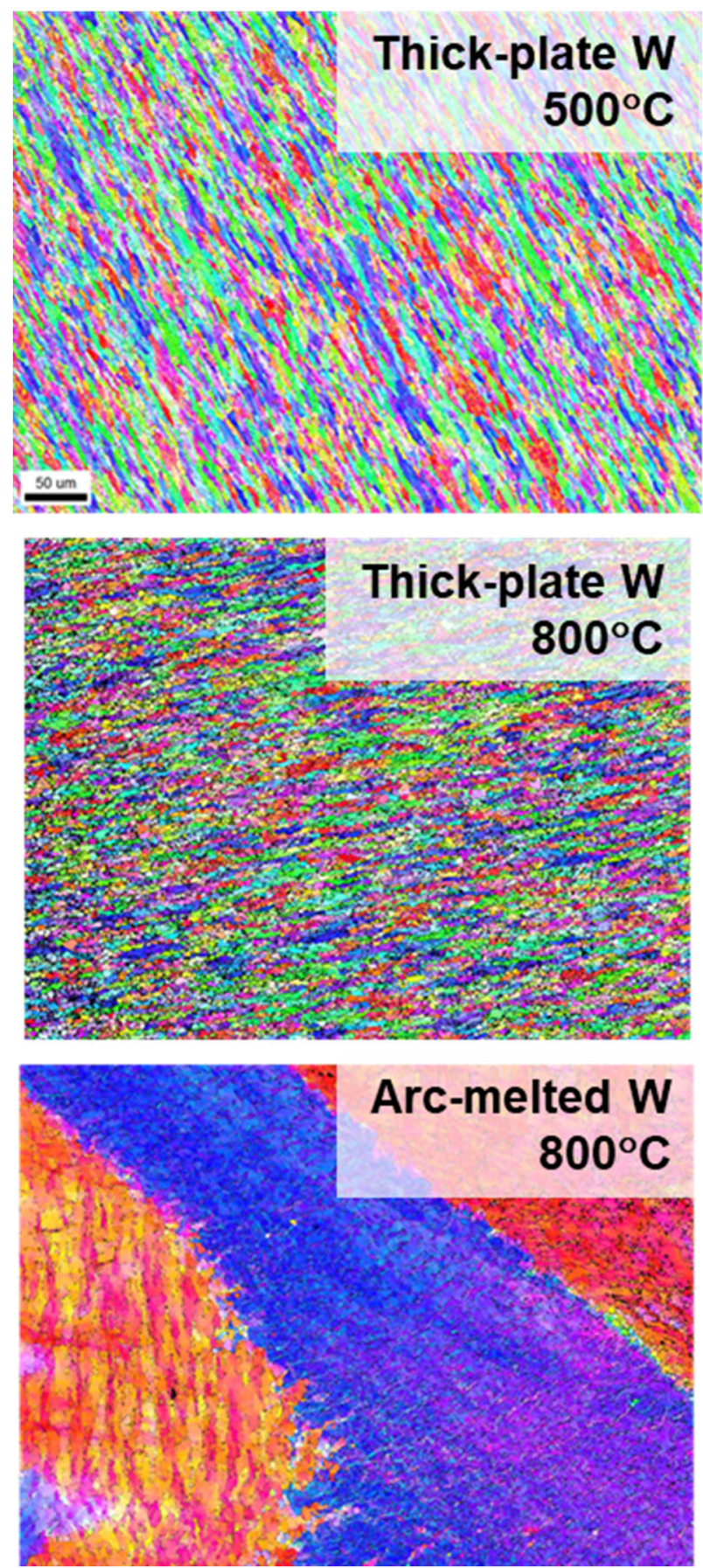

Figure 1. (left) EBSD grain maps of three of the neutron-irradiated $\mathrm{W}$ samples, indicating banded microstructures in the thick-plate material and a subgrain structure arising from large as-cast grain in the arc-melted material. Temperatures indicate the irradiated capsule target temperature.
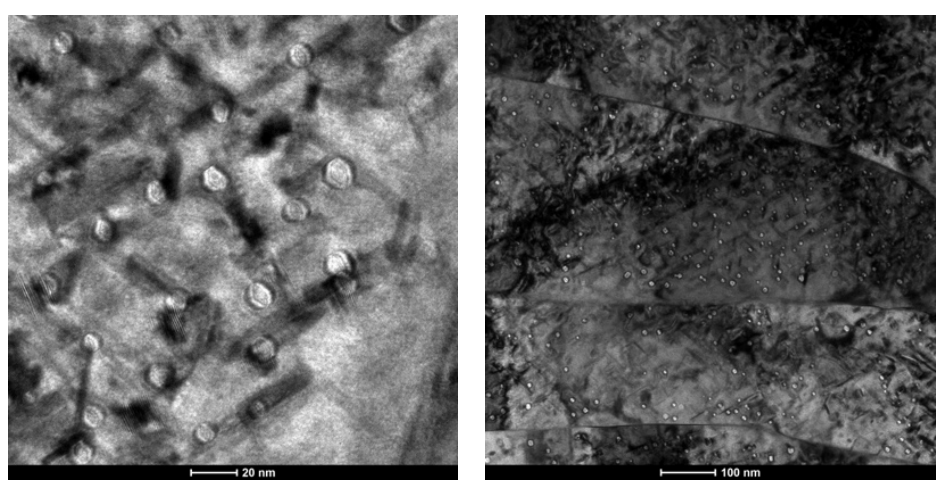

Figure 2. (above) TEM images of voids and precipitates in Gd-shielded irradiated tungsten.
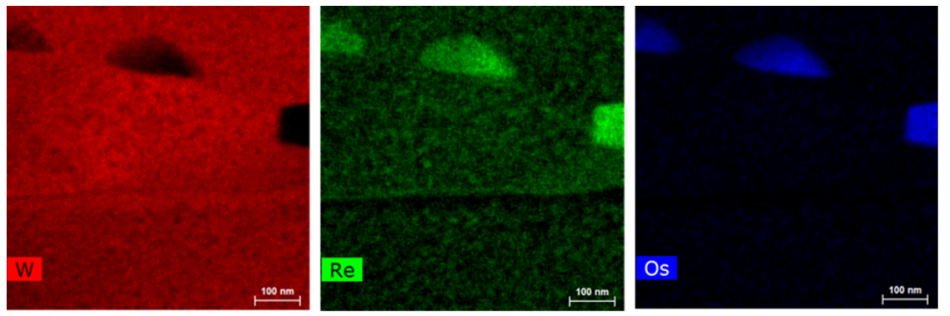

Figure 3. (above) STEM X-ray maps of W, Re, and $\mathrm{Os}$ in Gd-shielded irradiated tungsten. 